EPJ Web of Conferences 59, 17010 (2013)

DOI: $10.1051 /$ epjconf/20135917010

(C) Owned by the authors, published by EDP Sciences, 2013

\title{
Laser ion acceleration in the high laser energy and high laser intensity regimes
}

\author{
E. d'Humières ${ }^{\mathrm{a}}$, R. Capdessus and V.T. Tikhonchuk \\ Univ. Bordeaux-CEA-CNRS, CELIA UMR 5107, 33405 Talence, France
}

\begin{abstract}
New laser facilities able to deliver either ultra high energy short pulses or ultra high intensity pulses are being constructed and will open new and exciting opportunities for laser ion acceleration. The interaction of a high intensity short pulse with underdense, near-critical and overdense targets has been studied using 2D Particle-In-Cell simulations in these regimes. In the ultra high energy regime, proton beams with maximum energies of hundreds of $\mathrm{MeV}$ and a high number of high energy protons could be accelerated using thin solid foils or low density targets. In the ultra high intensity regime, radiation losses will start affecting laser ion acceleration using thin overdense targets for intensities higher than $10^{22} \mathrm{~W} / \mathrm{cm}^{2}$, and produce very energetic ions.
\end{abstract}

\section{INTRODUCTION}

In the last few years, intense research has been conducted on laser-accelerated ion sources and their applications [1]. Ion beams accelerated from solid planar targets have exceptional properties, i.e. high brightness and high spectral cut-off, high directionality and laminarity, and short burst duration $(\sim$ ps at the source). PETAL is a ultra high energy (UHE) multi-kJ Petawatt-class laser being built in France near Bordeaux [2]. Another new generation of laser systems such as the Apollon laser now being built in France will be able to deliver ultra high intensities (UHI) of the order of $10^{23} \mathrm{~W} / \mathrm{cm}^{2}$. These facilities open new and exciting opportunities for laser ion acceleration. The interaction of a high intensity short pulse with underdense, near-critical and overdense targets has been studied using 2D Particle-In-Cell simulations in these regimes. Section 2 is dedicated to the study of the interaction of a high intensity short pulse with ultra thin overdense targets using projected PETAL parameters. Section 3 presents the study of the interaction of a PETAL high intensity short pulse with underdense and near-critical density targets. In Section 4, simulations in the ultra high intensity regime show the effects of radiation losses on laser ion acceleration using thin overdense targets. Section 5 is dedicated to conclusions and perspectives.

\section{UHE REGIME: INTERACTION WITH THIN TARGETS}

The goal of this section is to study laser ion acceleration in the ultra high energy regime $(>1 \mathrm{~kJ})$. We have performed 2D Particle-In-Cell simulations with the code PICLS [3] to study the interaction of the expected PETAL laser pulse with a thin target in the transparency regime [4]. The wavelength of the incident pulse is $1 \mu \mathrm{m}$, its pulse duration is $500 \mathrm{fs}$ and its irradiance is $10^{21} \mathrm{~W} / \mathrm{cm}^{2}$. The FWHM of the focal spot is $30 \mu \mathrm{m}$. The p-polarized Gaussian pulse interacts with the target in normal incidence.

\footnotetext{
ae-mail: dhumieres@celia.u-bordeaux1.fr
}

This is an Open Access article distributed under the terms of the Creative Commons Attribution License 2.0, which permits unrestricted use, distribution, and reproduction in any medium, provided the original work is properly cited. 

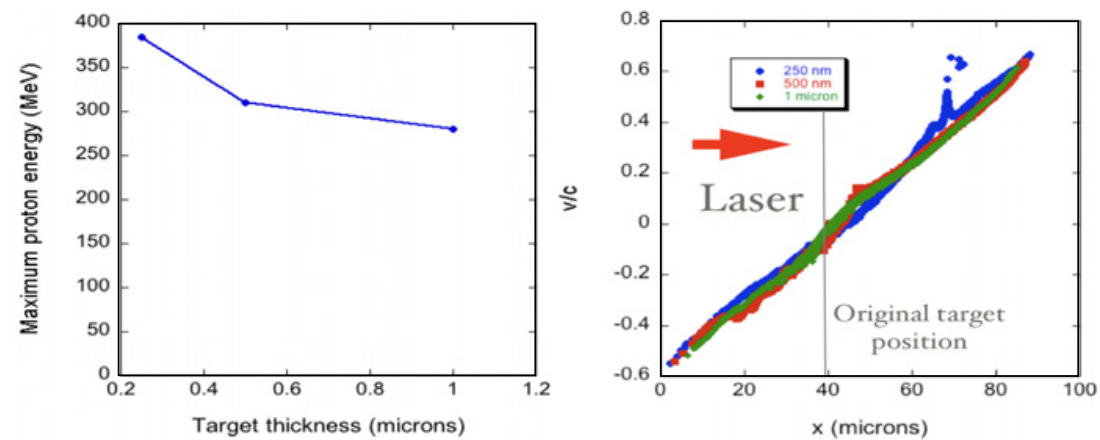

Figure 1. Variation of the maximum proton energy for target thicknesses: $250 \mathrm{~nm}, 500 \mathrm{~nm}$ and $1 \mu \mathrm{m}$ (a). Proton phase space for these three thicknesses $0.62 \mathrm{ps}$ after the beginning of the simulation (b).

The plasma is composed of protons and electrons with a constant $400 \mathrm{nc}$ density, where $\mathrm{n}_{\mathrm{c}}$ is the critical density. The plasma slab thickness was varied from 0.2 to $1 \mu \mathrm{m}$.

The maximum proton energy (MPE) measured in the simulation depends on target thickness and reaches several hundreds of MeV. Figure 1(a) shows the variation of the MPE when it saturates for three target thicknesses from $250 \mathrm{~nm}$ to $1 \mu \mathrm{m}$. Figure 1(b) shows the proton phase space after $0.62 \mathrm{ps}$ for these three cases and indicates that the most energetic protons come from the rear surface of the target. It is interesting to note that the thinnest target becomes transparent to the laser and a shock structure develops in the decreasing density gradient [5]. It will not be straightforward to accelerate protons with such thin targets without controlling the laser contrast. A simulation using a 10 microns $\mathrm{CH}$ target at solid density with a preplasma and similar laser parameters predicts a MPE of around $110 \mathrm{MeV}$. A plasma mirror or doubling the frequency could improve the contrast. With a $250 \mathrm{~nm}$ foil and at $2 \omega$, the MPE is lowered to $160 \mathrm{MeV}$ and $3.1 \times 10^{10}$ protons/MeV at $100 \mathrm{MeV}$ are expected. In this case, the laser energy is lowered to take into account the conversion efficiency and laser intensity is $5 \times 10^{20} \mathrm{~W} / \mathrm{cm}^{2}$. Other parameters are kept constant.

\section{UHE REGIME: INTERACTION WITH UNDERDENSE AND NEAR-CRITICAL TARGETS}

Another promising way to accelerate ions to high energies is to use underdense or near-critical density targets [6]. This approach has recently received a lot of attention experimentally [7] and theoretically $[5,8]$. Compared to solid targets where the laser energy absorption is surfacic, with low density targets, laser-plasma interaction is volumic leading to higher laser energy absorption. This acceleration regime also has several advantages for applications as less debris are produced at each laser shot, high repetition rate lasers can be used more easily and the laser contrast is also not as paramount. We have simulated the interaction of a laser pulse with a near-critical density target and an underdense target using laser parameters of Section 2 with PICLS in 2D. In both cases the plasma is composed of protons and electrons. For the first case, the target density is constant, $2 \mathrm{n}_{\mathrm{c}}$, and its thickness is $100 \mu \mathrm{m}$. For the underdense case, the target density profile is a cosine square with a full-width at half maximum of $100 \mu \mathrm{m}$ and a maximum density of $0.4 \mathrm{n}_{\mathrm{c}}$. A high laser absorption of $75.9 \%$ is measured in the $2 \mathrm{n}_{\mathrm{c}}$ case and $18.9 \%$ is measured in the $0.4 \mathrm{n}_{\mathrm{c}}$ case as the laser propagates through the plasma leaving a large channel behind. The generated hot electron population produces strong electrostatic fields at the back of the target. As explained in previous studies [8], the magnetic field plays a significant role in the ion acceleration process and focusing.

Figure 2 shows the proton phase space at the middle of the simulation box on the laser propagation axis $0.66 \mathrm{ps}$ after the beginning of the simulation (a), and $1.16 \mathrm{ps}$ after the beginning of the simulation 

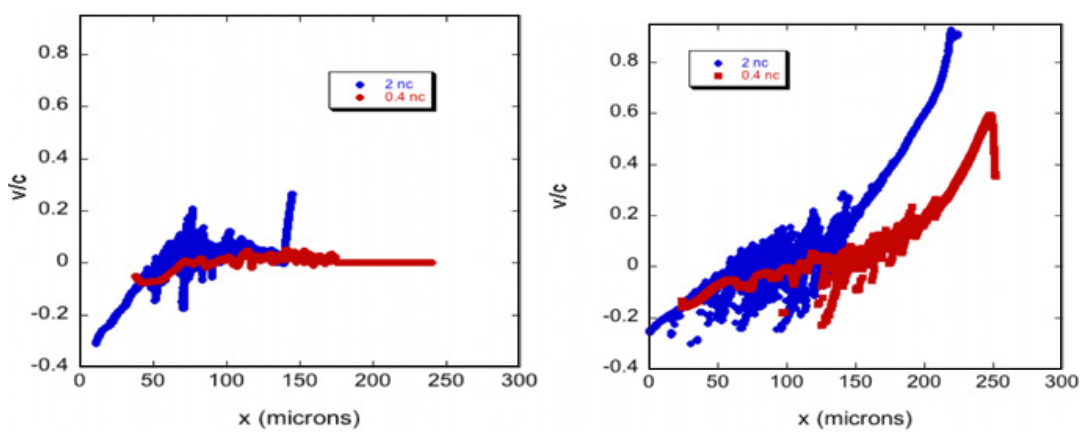

Figure 2. Proton phase space at the middle of the simulation box on the laser propagation axis $0.66 \mathrm{ps}$ after the beginning of the simulation (a), and $1.16 \mathrm{ps}$ after the beginning of the simulation (b).

(b). For both cases the most energetic protons come from a region close to the back surface of the target. Wave breaking is clearly visible in the underdense case as expected from previous theoretical studies. The MPE in the near-critical case is more than $400 \mathrm{MeV}$, which is comparable with the solid density target simulation shown in Figure 1 , and $7 \times 10^{9}$ protons $/ \mathrm{MeV}$ at $200 \mathrm{MeV}$ are expected. It reaches a little more than $200 \mathrm{MeV}$ in the underdense case with $2 \times 10^{10}$ protons $/ \mathrm{MeV}$ at $100 \mathrm{MeV}$.

Interaction conditions are not optimized. In this low density regime it is possible to find an optimum thickness and a key parameter controlling the acceleration process is the product of the density and the thickness. This optimum thickness also depends on laser parameters [5, 7, 8]. Interaction in this regime could therefore lead to even higher MPE than with solid targets. The near-critical target could be achieved experimentally using a foam or a foil exploded by a nanosecond pulse, the underdense target using a gas jet or also a foil exploded by a nanosecond pulse.

\section{UHI WITH AND WITHOUT SELF-FORCE}

The goal of this section is to study how laser ion acceleration using thin overdense targets evolves in the ultra high intensity regime $\left(>10^{22} \mathrm{~W} / \mathrm{cm}^{2}\right)$ and to assess the importance of radiation losses in this regime. The radiation losses model used is based on the so-called Sokolov model. Its implementation in PICLS is described in [9]. A set of simulations, selected to highlight both limits of the classical self-force regime and which can be compared with other published results $[9,10]$, is shown here.

In a first set of simulations, the wavelength of the incident pulse is $0.8 \mu \mathrm{m}$, its pulse duration is $21 \mathrm{fs}$ and its irradiance is $1.6 \times 10^{22} \mathrm{~W} / \mathrm{cm}^{2}$. The FWHM of the focal spot is $5 \mu \mathrm{m}$. The p-polarized Gaussian pulse interacts with the target in normal incidence. The plasma is composed of protons and electrons with a constant $400 n_{c}$ density. The plasma slab thickness was varied from 0.2 to $0.8 \mu$ m. Figure 3(a) shows the simulated proton spectra with and without radiation losses for the $0.25 \mu \mathrm{m}$ case. In this range of thicknesses, radiation losses have a measurable effect on ion acceleration but this effect remains small. In a second set of simulations, the wavelength of the incident pulse is $0.8 \mu \mathrm{m}$, its pulse duration is $48 \mathrm{fs}$ and its irradiance is $1.5 \times 10^{23} \mathrm{~W} / \mathrm{cm}^{2}$. The FWHM of the focal spot is $5 \mu \mathrm{m}$. The circularly-polarized trapezoidal pulse interacts with the target in normal incidence. The plasma is composed of deuterons and electrons with a constant $10 \mathrm{n}_{c}$ density. The plasma slab thickness was varied from 0.4 to $5 \mu \mathrm{m}$. Figure 3(b) shows the simulated deuteron spectra with and without radiation losses for the $5 \mu \mathrm{m}$ case. For such a high intensity the radiation losses effects on ion acceleration become important but very high ion energy are nevertheless obtained.

It is interesting to note that the number of Deuteron ions in the range from 500 to $1300 \mathrm{MeV}$ is more important in the case where the self-force is taken into account. This is due to the fact that the electron energy spectrum is also shifted to lower energies with a number of accelerated electrons very close in 

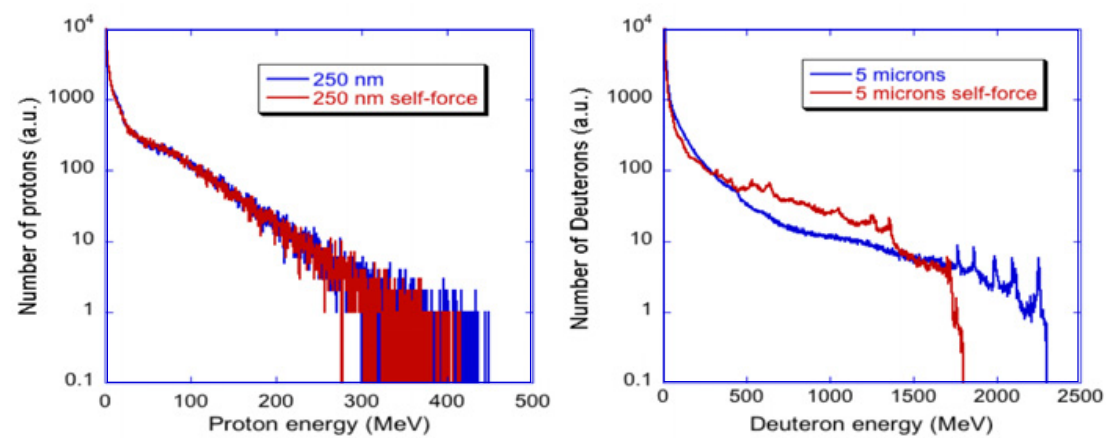

Figure 3. (a) Proton spectra for the $1.6 \times 10^{22} \mathrm{~W} / \mathrm{cm}^{2}$ pulse interacting on a $250 \mathrm{~nm}$ high density foil. (b) Deuteron spectra for the $1.5 \times 10^{23} \mathrm{~W} / \mathrm{cm}^{2}$ pulse interacting on a $5 \mu \mathrm{m}$ overdense foil. Blue curve: without radiation losses. Red curve: with radiation losses.

both cases. The energy transfer between electrons and ions therefore still leads to a high number of Deuteron ions in the self-force case, but at lower energies. Another interesting feature is the presence of peaks in the high energy part of the Deuteron ions spectrum when taking into account the self-force. These bunch-like structures will need to be investigated in a future study.

\section{CONCLUSIONS AND PERSPECTIVES}

The interaction of a high intensity short pulse with underdense, near-critical and overdense targets has been studied in the UHE and in the UHI regimes to estimate ion acceleration possibilities on future laser facilities. This study has been achieved using the Particle-In-Cell code PICLS in which charged particles energy losses through radiation have been implemented. In the ultra high energy regime, proton beams with MPE of several hundreds of MeV and a high number of high energy protons could be accelerated using thin solid foils or underdense and near-critical targets. In the ultra high intensity regime, radiation losses will start affecting laser ion acceleration using thin overdense targets for intensities higher than $10^{22} \mathrm{~W} / \mathrm{cm}^{2}$, but MPE of a few hundreds $\mathrm{MeV}$ can still be reached. A more systematic study of the influence of radiation losses on the various high intensity ion laser acceleration mechanisms is now needed and more optimization of these regimes should be done.

This work was partially supported by EURATOM within the "Keep-in-Touch" activities, by the Conseil Régional d'Aquitaine (MAPI project) and by the GENCI allocation c2011-056129.

\section{References}

[1] J. Fuchs et al., Nature Physics 2, 48 (2006), L. Robson et al., Nat. Phys. 3, 58 (2007)

[2] N. Blanchot et al., Plasma Phys. Control. Fusion 50, 124045 (2008)

[3] Y. Sentoku and A. Kemp, Journal of Computational Physics 227, 6846 (2008)

[4] Q. Dong et al., Phys. Rev. E 68, 026408 (2003), E. d'Humières et al., Phys. Plasmas 12, 062704 (2005)

[5] E. d'Humières et al., J. Phys.: Conf. Ser. 244, 042023 (2010).

[6] T. Zh. Esirkepov et al., JETP Lett. 70, 82 (1999), M. Yamagiwa et al., Phys. Rev. E 60, 5987 (1999), Y. Sentoku et al., Phys. Rev. E 62, 7271 (2000) 


\section{IFSA 2011}

[7] L. Willingale et al., Phys. Rev. Lett. 96, 245002 (2006), A. Yogo et al., Phys. Rev. E 77, 016401 (2008), P. Antici et al., New J. Phys. 11, 023038 (2009)

[8] S. V. Bulanov et al., Phys. Rev. Lett. 98, 049503 (2007), L. Willingale et al., Phys. Rev. Lett. 98, 049504 (2007)

[9] R. Capdessus et al., Phys. Rev. E. 86, 036401(2012)

[10] T. Schlegel et al., Phys. Plasmas 16, 081303 (2009), M. Tamburini et al., New Journal of Physics 12, 123005 (2010) 